TITLE:

\title{
Direct numerical simulation of turbulent channel flow under a uniform magnetic field for large- scale structures at high Reynolds number
}

\section{AUTHOR(S):}

Satake, Shin-ichi; Kunugi, Tomoaki; Takase, Kazuyuki; Ose, Yasuo

\section{CITATION:}

Satake, Shin-ichi ... [et al]. Direct numerical simulation of turbulent channel flow under a uniform magnetic field for large-scale structures at high Reynolds number. PHYSICS OF FLUIDS 2006, 18(12): 125106.

\section{ISSUE DATE:}

2006-12

URL:

http://hdl.handle.net/2433/39789

\section{RIGHT:}

Copyright 2006 American Institute of Physics. This article may be downloaded for personal use only. Any other use requires prior permission of the author and the American Institute of Physics. 


\title{
Direct numerical simulation of turbulent channel flow under a uniform magnetic field for large-scale structures at high Reynolds number
}

\author{
Shin-ichi Satake \\ Department of Applied Electronics, Tokyo University of Science, 2641 Yamazaki, Noda, \\ Chiba 278-8510, Japan \\ Tomoaki Kunugi \\ Department of Nuclear Engineering, Graduate School of Engineering, Kyoto University, \\ Yoshida, Sakyo, Kyoto 606-8501, Japan \\ Kazuyuki Takase \\ Japan Atomic Energy Agency, Tokai Naka, Ibaragi 319-1195, Japan \\ Yasuo Ose \\ Yamato System Engineer, 1-17-1 Hitachi, Ibaraki 317-0063, Japan
}

(Received 10 May 2006; accepted 2 November 2006; published online 15 December 2006)

\begin{abstract}
A direct numerical simulation (DNS) of turbulent channel flow with high Reynolds number has been carried out to show the effects of the magnetic field. In this study, the Reynolds number for channel flow based on bulk velocity $U_{b}$, viscosity $\nu$, and channel width $2 \delta$ was set to be constant; $\operatorname{Re}_{b}=2 \delta U_{b} / \nu=45818$. A uniform magnetic field was applied in the direction of the wall normal. The value of the Hartmann number, Ha were 32.5 and 65 , where $\mathrm{Ha}=2 \delta B_{0} \sqrt{\sigma / \rho \nu}$. The turbulent quantities such as the mean flow, turbulent stress, and turbulent statistics were obtained by DNS. Although the influence of the magnetohydrodynamic dissipation terms in the turbulent kinetic energy budget was small, large-scale turbulent structures, e.g., vertical structures, low-speed streaks, ejection, and sweep, were found to decrease at the central region of the channel. Consequently, the difference between production and dissipation in the turbulent kinetic energy decreased with increasing Hartmann number at the central region and large-scale structures at this region were reduced. (C) 2006 American Institute of Physics. [DOI: 10.1063/1.2404943]
\end{abstract}

\section{INTRODUCTION}

In recent developments in nuclear fusion research, certain design concepts for liquid breeder blankets for nuclear fusion reactors use molten salts, such as FLiBe, as coolant material. ${ }^{1}$ The mean velocity of liquid coolant material in a reactor is strongly influenced by the magnetic field, and hence it is important to clarify the turbulent magnetohydrodynamic (MHD) flow behavior for an applied magnetic field perpendicular to the main flow. Furthermore, because the flow characteristics of the coolant at high Reynolds number ${ }^{2}$ are assumed to be different from the usual turbulent MHD flow, it is important to investigate high-Reynolds number flow under a magnetic field for the design of real application facilities.

Extensive experimental work has been done by Reed and Lykoudis $^{3}$ for turbulent channel flow in a transverse magnetic field. The first numerical work on large eddy simulations (LES) of MHD channel flow was performed by Shimomura, ${ }^{4}$ who studied the drag increase due to the Hartmann effect under a uniform wall-normal magnetic field; his results were in good agreement with the results presented by Reed and Lykoudis. ${ }^{3}$ Direct numerical simulations (DNS) for turbulent pipe flow were made by Orlandi ${ }^{5}$ and Satake et al. ${ }_{7}{ }_{7}$ for open channel flow the study was done by Satake et al. ${ }^{7}$ and for turbulent channel flow the work was done by Lee and Choi. ${ }^{8}$ A DNS database is necessary for making turbulence models such as the $k$-e model, ${ }^{9}$ second-moment closer model, ${ }^{10}$ and large eddy simulation. ${ }^{11}$ These models require that velocity-electric correlation terms be determined. Kenjeres and Hanjalic ${ }^{10}$ performed turbulence modeling using estimations of these terms from the DNS database. When a flow exhibits a high Reynolds number, large-scale turbulent structures appear at the channel center region, as was found in experimental works ${ }^{12}$ and DNS. ${ }^{13-16}$ The structures are clustered at the core region where they form hierarchical structures. ${ }^{12}$ These large structures cannot be ignored and therefore it is very important to investigate their fundamental characteristics of these structures. However, at present very little is known about the behavior of these large-scale structures under a magnetic field; it is not even certain whether they continue to be existent.

In this paper, we apply our channel DNS database ${ }^{14}$ at high Reynolds number to uniform magnetic fields. The objectives of this study are to understand the large-scale reduction of the turbulent channel flow in a transverse magnetic field and to reveal the mechanism of the decrease of these large-scale turbulence structures.

\section{NUMERICAL FORMULATION}

\section{A. Governing equations}

The governing equations at low magnetic Reynolds number are written in their dimensional forms:

$$
\nabla \cdot \mathbf{u}=0
$$


TABLE I. Number of grid points, Reynolds number, and grid resolution.

\begin{tabular}{cccccc}
\hline \hline $\operatorname{Re} \tau$ & Ha & Grid number & $\Delta x+$ & $\Delta y+$ & $\Delta z+$ \\
\hline 1120 & 0 & $1024 \times 1024 \times 768$ & 17.2 & $0.163-4.25$ & 9.16 \\
1150 & 32.5 & $1024 \times 1024 \times 768$ & 17.6 & $0.167-4.4$ & 9.4 \\
1194 & 65 & $1024 \times 1024 \times 768$ & 18.2 & $0.17-4.54$ & 9.6 \\
\hline \hline
\end{tabular}

$$
\begin{aligned}
& \frac{\partial \mathbf{u}}{\partial t}+\mathbf{u} \cdot \nabla \mathbf{u}=-\nabla \cdot p+\nu \nabla^{2} \mathbf{u}+\frac{1}{\rho} \mathbf{j} \times \mathbf{B}, \\
& \mathbf{j}=\sigma(\mathbf{E}+\mathbf{u} \times \mathbf{B}), \\
& \nabla \cdot \mathbf{j}=0, \\
& \nabla \times \mathbf{E}=0,
\end{aligned}
$$

where $\mathbf{u}$ is the velocity, $p$ is the pressure divided by density, $\nu$ is the kinetic viscosity, $\rho$ is the density, $\mathbf{j}$ is the current density, $\mathbf{B}$ is the magnetic field, $\sigma$ is the electrical conductivity, and $\mathbf{E}$ is the electric field. The induced magnetic field at low magnetic Reynolds number is small compared to the applied magnetic field. The magnetic field in the present study is demoted by $\mathbf{B}_{0}$, a constant. The electric field is described using the electrical potential $\phi$ as $\mathbf{E}=-\nabla \phi$. Thus, we have the following nondimensional equations:

$$
\nabla \cdot \mathbf{u}=0
$$

$$
\begin{aligned}
\frac{\partial \mathbf{u}}{\partial t}+\mathbf{u} \cdot \nabla \mathbf{u}= & -\nabla \cdot p+\frac{1}{\operatorname{Re}_{b}} \nabla^{2} \mathbf{u} \\
& +\frac{\mathrm{Ha}^{2}}{\operatorname{Re}_{b}}\left[\left(-\nabla \phi+\mathbf{u} \times \mathbf{B}_{0}\right) \times \mathbf{B}_{0}\right],
\end{aligned}
$$

$$
\nabla^{2} \phi=\nabla \cdot\left(\mathbf{u} \times \mathbf{B}_{0}\right)=\mathbf{B}_{0} \cdot \boldsymbol{\omega},
$$

where $\boldsymbol{\omega}$ is the vorticity expressed as $\boldsymbol{\omega}=\nabla \times \mathbf{u}$. The above equations are normalized by channel width $2 \delta$ and bulk velocity $u_{b}=\frac{1}{2 \delta} \int_{0}^{2 \delta}\langle u\rangle d y$. Here the symbol \langle\rangle denotes an average in the $x-z$ plane. The Hartmann number $\mathrm{Ha}=2 \delta B_{0} \sqrt{\sigma / \rho \nu}$. The bulk Reynolds number $\operatorname{Re}_{b}=2 \delta u_{b} / \nu$.

\section{B. Computational conditions}

Our DNS code is a hybrid of spectral finite difference methods. ${ }^{14,17}$ The spectral method is used to compute the spatial discretization in the stream and spanwise directions.

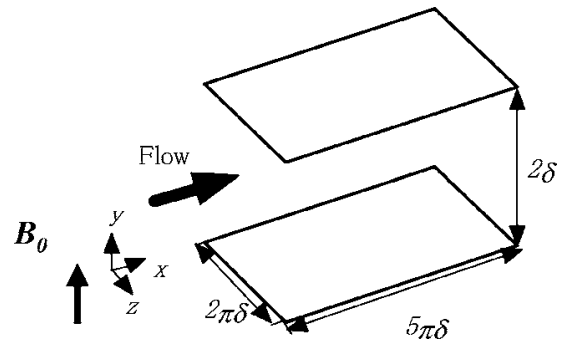

FIG. 1. Computational domain.

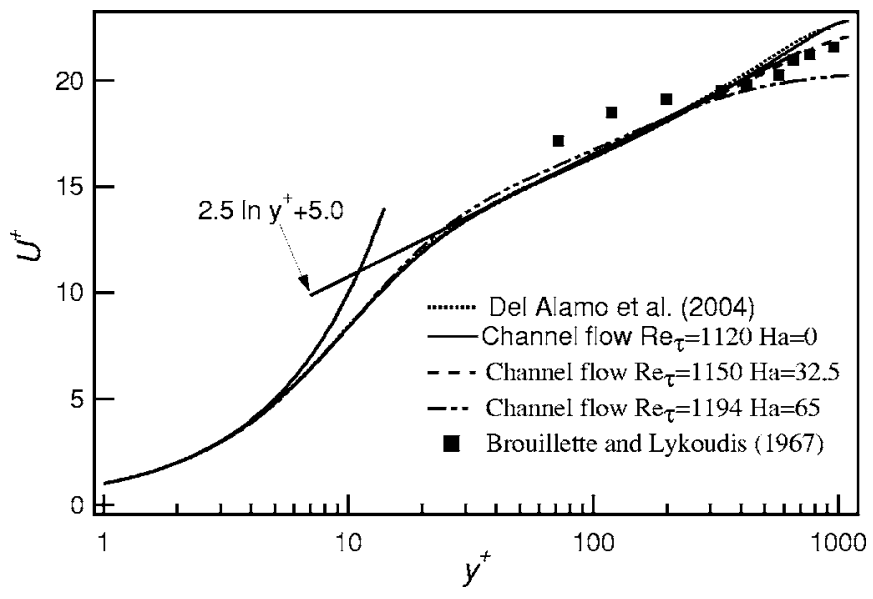

FIG. 2. Mean velocity profiles.

Nonlinear terms are calculated in physical space and the grid used to compute the nonlinear terms has a resolution 1.5 times finer in these directions to remove aliasing errors. The derivative in the wall normal direction is computed by a second-order finite difference scheme at staggered grid arrangements. The stream and spanwise components are located at the pressure point. The wall normal component is shifted to half the mesh size. The scalar potential for the electrical field is also located at the pressure point. The Helmholtz equation for viscous terms and the pressure Poisson equations are solved by using a tridiagonal matrix technique in Fourier space. The scalar potential is also solved by the same algorithm. These equations in time are integrated by using the fractional-step method. The second-order Crank-Nicholson scheme applied to the viscous terms is treated implicitly, whereas a modified third-order RungeKutta scheme is used for the nonlinear and Lorentz force terms explicitly. The number of grid points, the Reynolds number $\operatorname{Re}_{\tau}=\delta u_{\tau} / \nu$ based on the channel half-width $\delta$ and friction velocity $u_{\tau}$, and the grid resolutions are summarized in Table I. Our computations are adopted for 224 GB main memory with 32 processor elements (PEs) on vector-parallel computers, Fujitsu VPP 5000 at JAEA and SX-7 at NIFS. The computational time per one step was $64 \mathrm{~s}$ and $47 \mathrm{~s}$ in

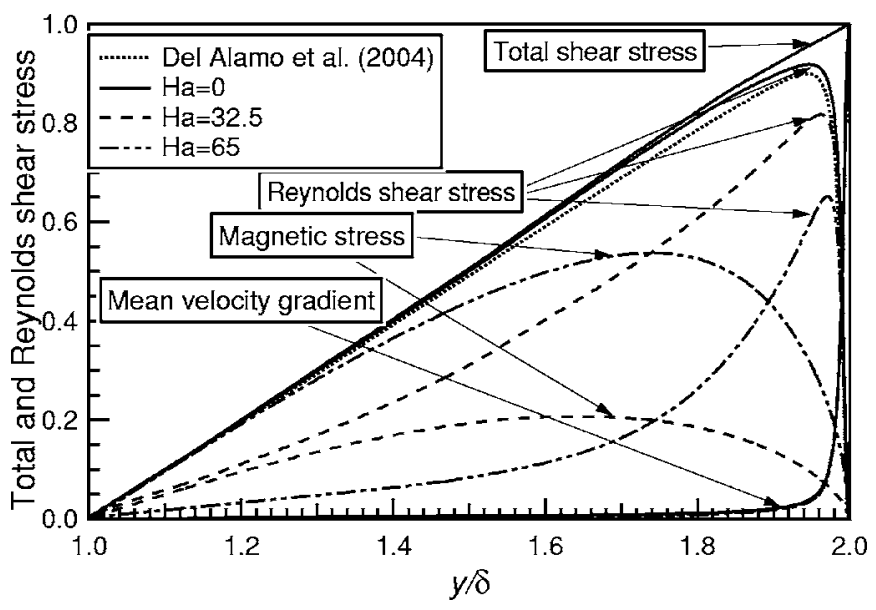

FIG. 3. Total and Reynolds shear stresses. 
(a)

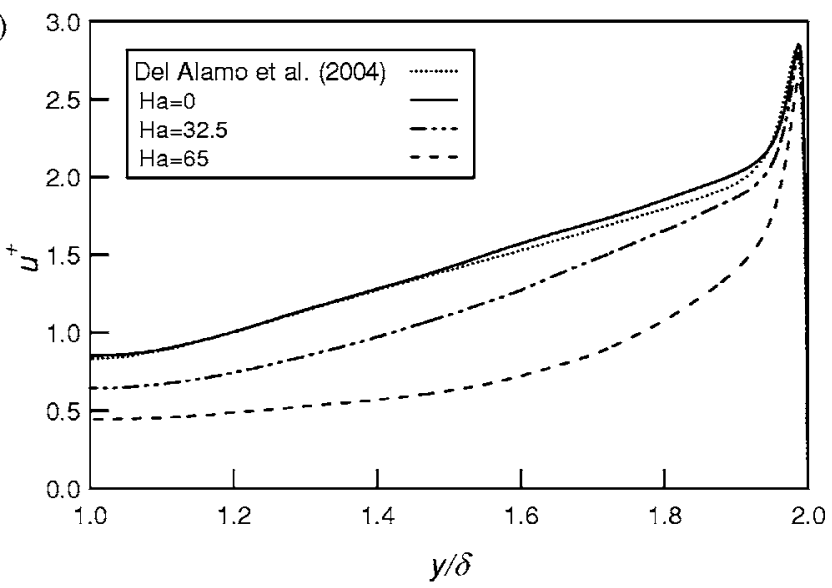

(b)

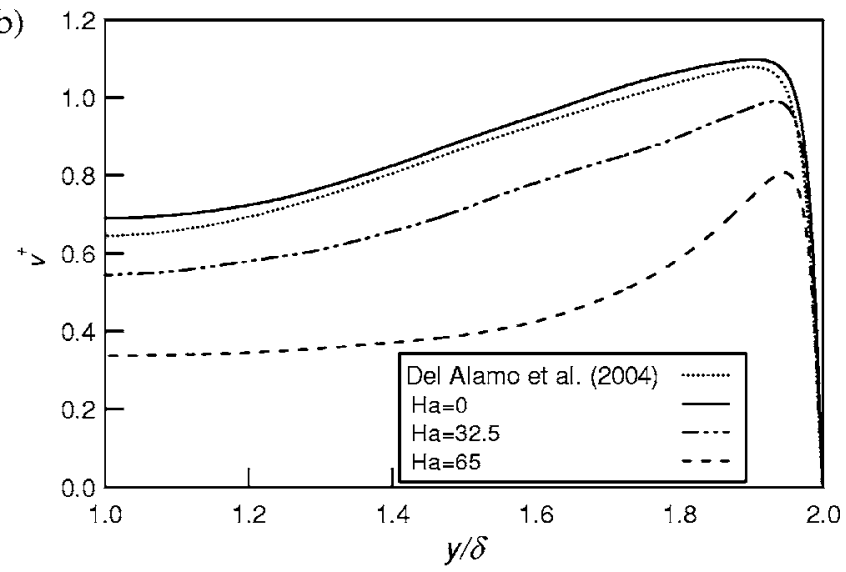

(c)

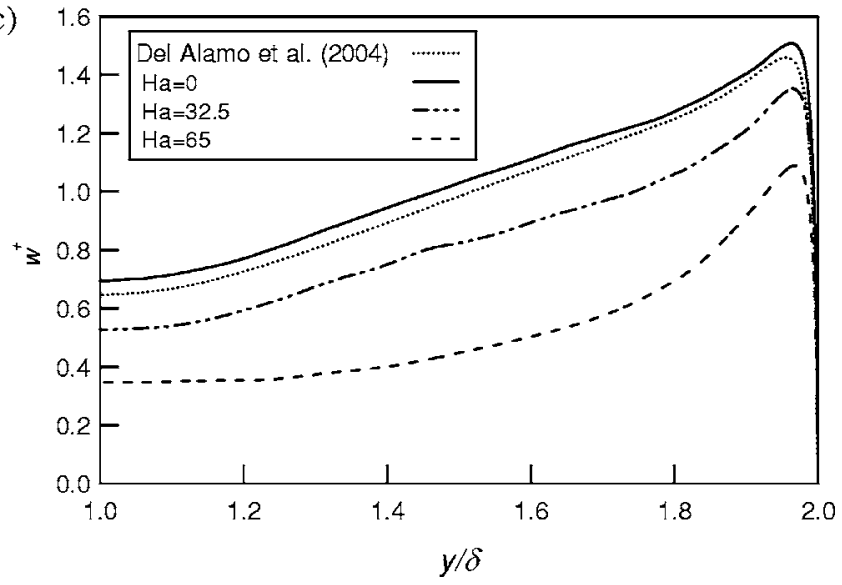

FIG. 4. Velocity fluctuation profiles.

SX-7 and VPP 5000, respectively. Total computational time for statistical averaging was $11.7\left(u_{b} T / 5 \pi \delta\right)$ after 100 $\left(u_{b} T / 5 \pi \delta\right)$ spend for becoming of the fully developing state from initial state. Periodic boundary conditions were applied to the streamwise $(x)$ and spanwise $(z)$ directions. For the wall-normal direction $(y)$, a nonuniform mesh spacing was employed that was specified by a hyperbolic tangent function. A nonslip condition at the wall was applied to the velocity components.

The numbers of computational grids used in this study were 1024, 1024, and 768 in the $x, y$, and $z$ directions, respectively. The uniform magnetic field $\mathbf{B}_{0}$ defined the $y$ axis

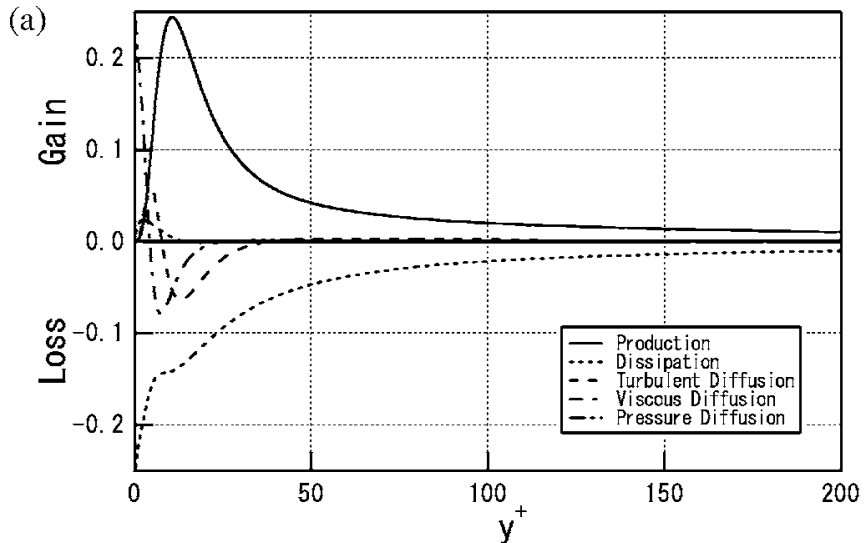

(b)

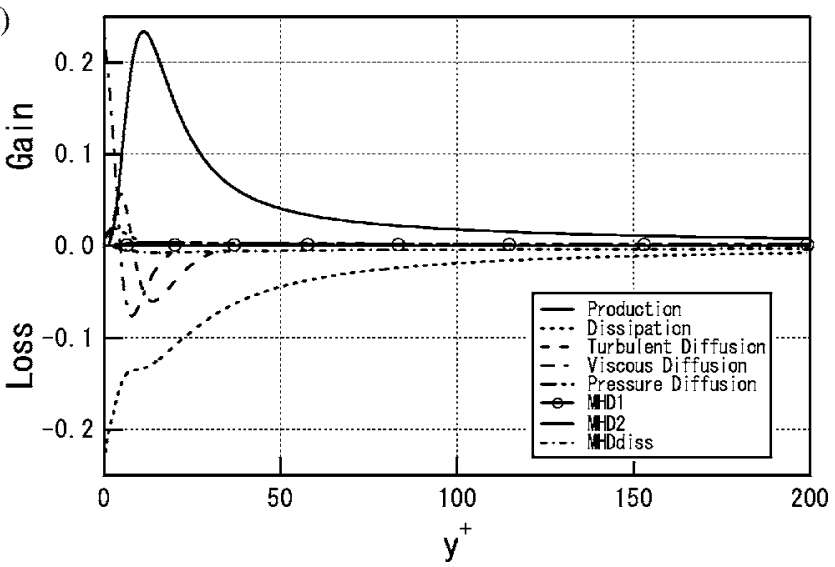

(c)

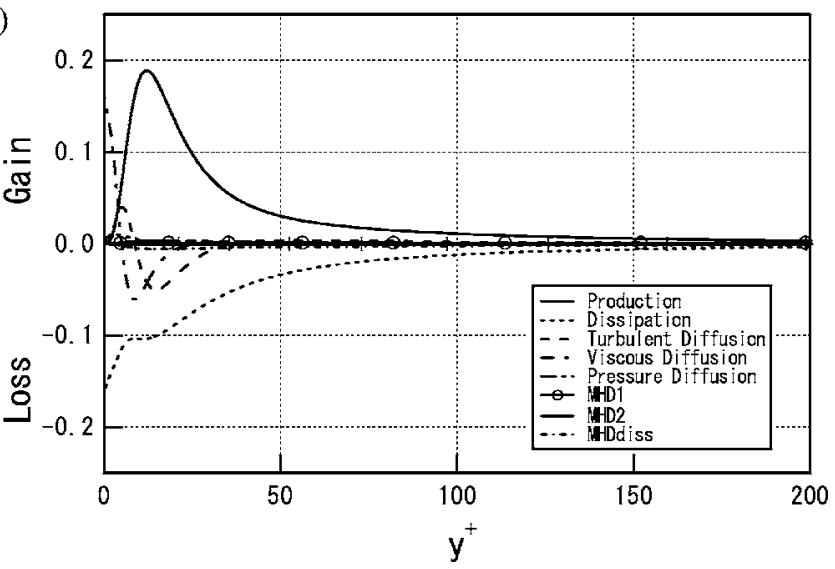

FIG. 5. Turbulent kinetic energy budget: (a) $\mathrm{Ha}=0$, (b) $\mathrm{Ha}=32.5$, (c) $\mathrm{Ha}=65$.

that lay along the axis of the streamwise direction in Fig. 1. The bulk Reynolds number was 45818; this number was based on the bulk velocity and channel width $2 \delta$. The fluid flowed with a constant mass flux. The Neumann condition for the electrical potential was adopted at the wall, i.e., an insulation wall assumption was applied here. The Hartmann numbers $\mathrm{Ha}=2 \delta B_{0} \sqrt{\sigma / \rho \nu}$ were set to 32.5 and 65 based on the magnetic field $B_{0}$, the kinematic viscosity $\nu$, the electrical conductivity $\sigma$, and the channel width $2 \delta$. Thereafter, $u, v$, and $w$ denoted the velocity components in the $x, y$, and $z$ directions, respectively. A subscript (+) represented a quantity that was nondimensionalized with $u_{\tau}$ and $\nu$. 


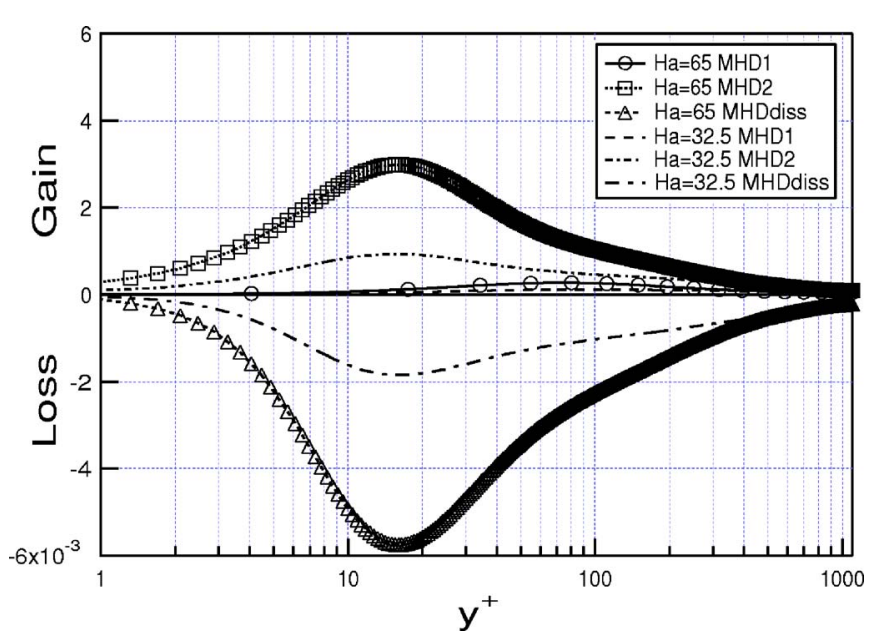

FIG. 6. MHD dissipation for MHD cases.

\section{RESULTS AND DISCUSSION}

Mean velocity profiles normalized by friction velocity are shown in Fig. 2. Satake et al. ${ }^{14}$ and Abe et al. ${ }^{15}$ reported that for high Reynolds number with no magnetic field, a logarithmic profile exists that is elongated at the channel center. The DNS profile of Del Alamo et al. ${ }^{13}\left(\operatorname{Re}_{\tau}=934\right)$ is also shown in Fig. 2. Their Reynolds number is close to our Reynolds number. The logarithmic profile of Del Alamo et $a .^{13}$ is in good agreement with that of our findings. Thus, the numerical accuracy for our calculation without a magnetic field is good. The experimental profile of Brouillette and Lykoudis ${ }^{18}(\mathrm{Re}=29000, \mathrm{Ha}=52.5)$ is also shown in Fig. 2. The logarithmic profile at $\mathrm{Ha}=65$ slightly increases at the channel center, and the wake region clearly becomes smaller. Although the logarithmic profile at $\mathrm{Ha}=32.5$ is maintained, the wake region decays. This indicates that the increase at the logarithmic region is primarily caused by the so-called
"Hartmann effect." That is, the Hartmann effect flattens the velocity profile, causing it to decrease with increasing Hartmann number. At $\mathrm{Ha}=65$, the profile becomes more rounded than at $\mathrm{Ha}=0$. The profile is in good agreement with the experimental profile reported by Brouillette and Lykoudis. ${ }^{18}$ A similar observation was made for DNS of channel flow ${ }^{8}$ and for experimental data of channel flow ${ }^{3,14}$ with a wall normal magnetic field.

Figure 3 shows the Reynolds shear stress, magnetic stress, and the total stress. The total shear stress is written as

$$
\frac{\partial U^{+}}{\partial y^{+}}-\overline{u^{+} v^{+}}+\frac{\mathrm{Ha}^{* 2}}{\operatorname{Re}_{\tau}^{2}} \int_{0}^{y^{+}}\left(u_{b}^{+}-U^{+}\right) d y^{+}=1-\frac{y^{+}}{\operatorname{Re}_{\tau}}
$$

where $\mathrm{Ha}^{*}=\delta B_{0} \sqrt{\sigma / \rho \nu}$ based on the channel half-width $\delta$. The total shear stress consists of the mean velocity gradient, Reynolds shear stress, and magnetic stress; and it becomes a straight line when the flow state is fully developed. The Reynolds stress profile of Del Alamo et al. ${ }^{13}$ is also shown in Fig. 3. Our calculation without a magnetic field is also highly accurate. At $\mathrm{Ha}=32.5$ and 65 , magnetic stress appears and cannot be ignored. Although magnetic stress exists in the whole of the channel at $\mathrm{Ha}=32.5$, the Reynolds shear stress is still dominant. On the other hand, at $\mathrm{Ha}=65$, the magnetic stress is very large, for the near region.

The velocity fluctuations are shown in Figs. 4(a)-4(c). The velocity fluctuations profile of Del Alamo et al. ${ }^{13}$ is also shown in Figs. 4(a)-4(c). Although our Reynolds number is slightly larger than that reported by Del Alamo et al. ${ }^{13}$ our profiles have a similar tendency compared to their results. Interestingly, all the components decrease at the channel center. The streamwise component shown in Fig. 4(a) remains near the wall region. The wall-normal component at $\mathrm{Ha}$ $=65$ in Fig. 4(b) is slightly shifted towards the region near the wall compared to the non-MHD case. In Fig. 4(c), the spanwise component at $\mathrm{Ha}=0$ shows a bulge. This phenom- (a)

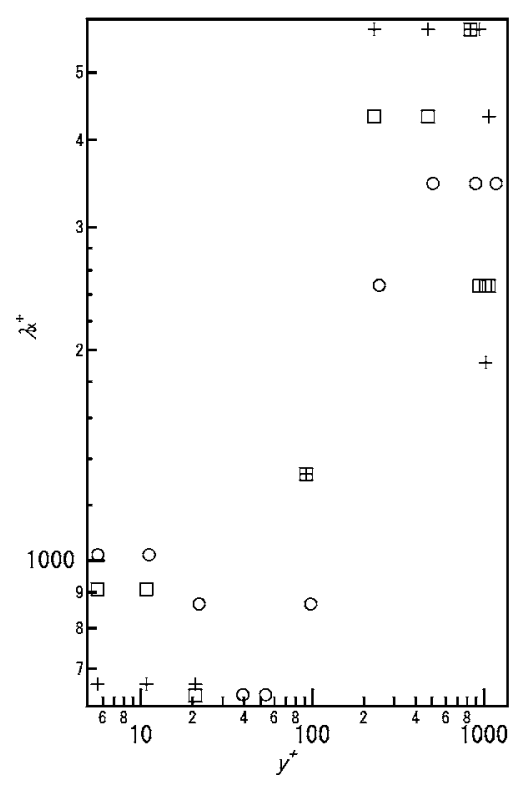

(b)

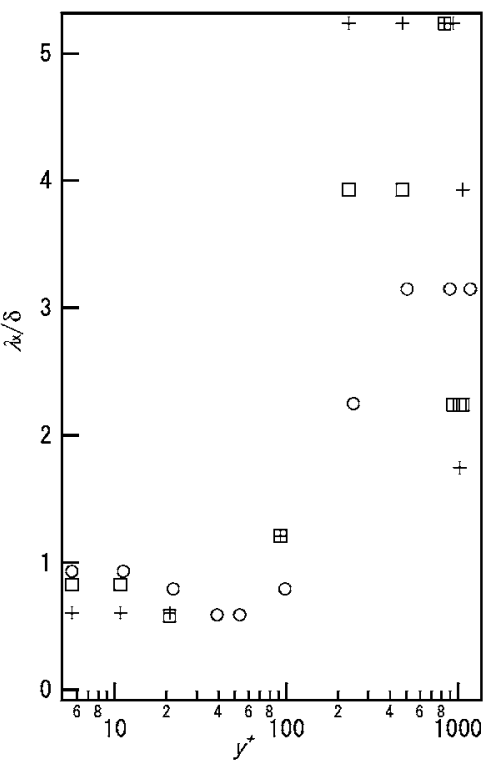

(c)

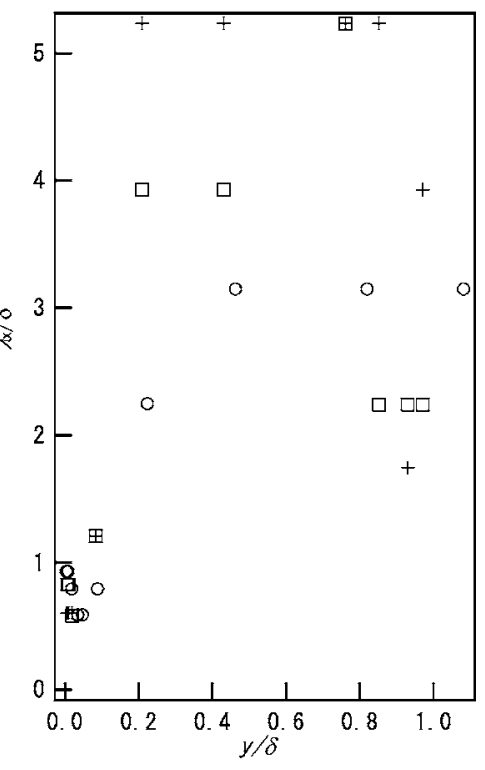

FIG. 7. Streamwise wavelength of the peak of the premultiplied power spectrum at different distances from the wall; $\bigcirc: \mathrm{Ha}=65, \square: \mathrm{Ha}=32.5,+\mathrm{Ha}=0$; (a) inner-inner scaling, (b) outer-inner scaling, (c) outer-outer scaling. 

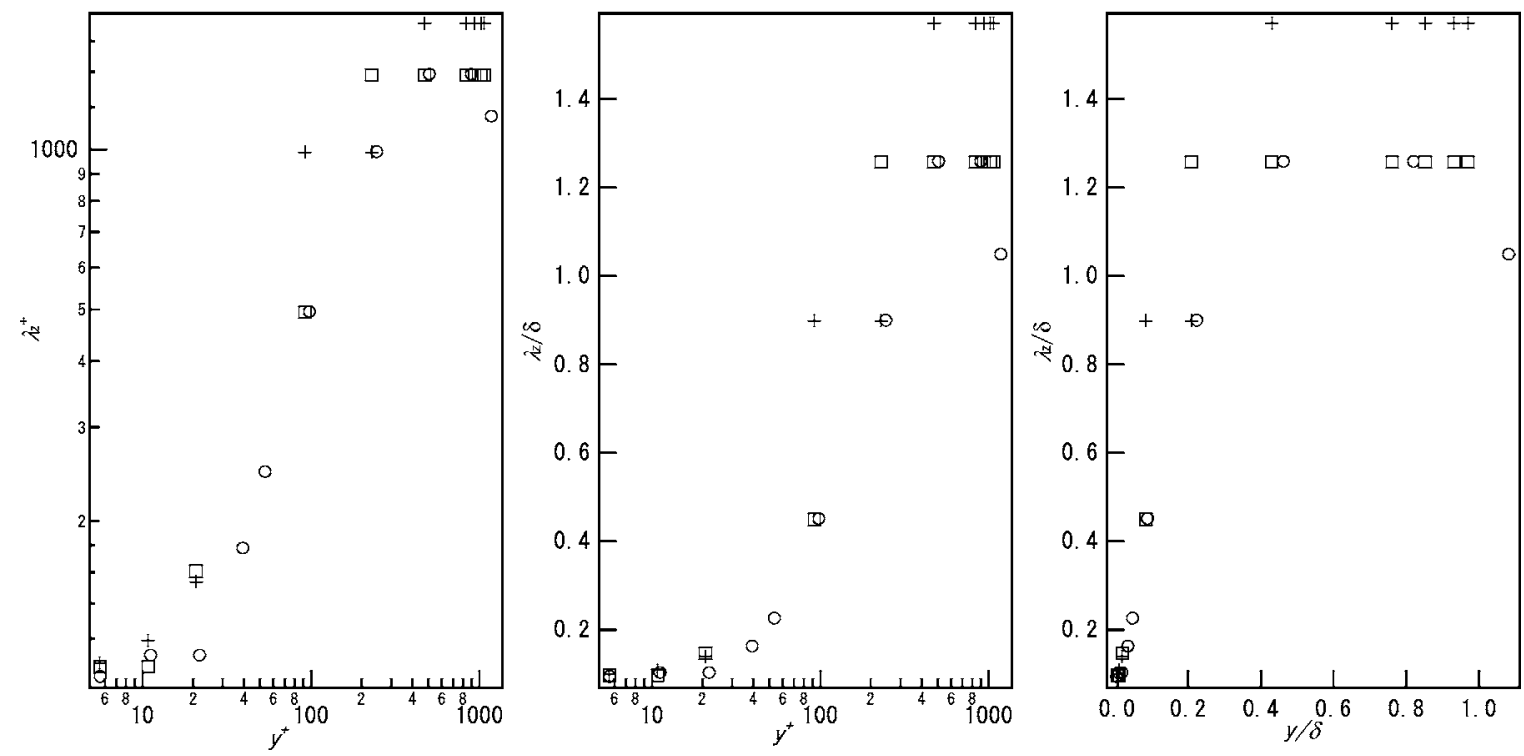

FIG. 8. Spanwise wavelength of the peak of the premultiplied power spectrum at different distances from the wall; $\bigcirc: \mathrm{Ha}=65, \square: \mathrm{Ha}=32.5,+: \mathrm{Ha}=0 ;(\mathrm{a})$ inner-inner scaling, (b) outer-inner scaling, (c) outer-outer scaling.

enon is seen for high Reynolds number flow. And also, DNS exhibits the phenomenon for other high Reynolds number. At $\mathrm{Ha}=32.5$ and 65 , the bulge decreases. This tendency for the velocity fluctuations to decay is also seen in the DNS of channel flow ${ }^{14}$ and experimental channel flows. ${ }^{3}$ Thus, this phenomenon brings about the decay of turbulent kinetic energy under a magnetic field. In order to understand this mechanism, we now discuss the turbulent kinetic energy budget level.

The turbulent kinetic energy budget is expressed as

$$
\begin{aligned}
0= & -\underbrace{-\overline{u^{\prime+} v^{\prime+}} \frac{\partial U^{+}}{\partial y^{+}}}_{\text {Production }}-\underbrace{\frac{1}{2} \frac{\partial \overline{u_{i}^{\prime+} u_{i}^{\prime+} v^{\prime+}}}{\partial y^{+}}}_{\text {Turbulent diffusion }}+\underbrace{\frac{\partial^{2} k}{\partial y^{+2}}}_{\text {Molecular diffusion }} \\
& \underbrace{-\frac{\partial}{\partial y^{+}}\left(\overline{v^{\prime+} p^{\prime+}}\right)}_{\text {Pressure diffusion }}-\underbrace{\frac{\overline{\partial u_{i}^{\prime+} \partial u_{i}^{\prime+}}}{\partial x_{j}^{+} \partial x_{j}^{+}}}_{\text {Dissipation }}-\underbrace{-\mathrm{Ha}^{2} w^{\prime+\frac{\partial \varphi^{+}}{\partial x^{+}}}}_{\text {MHDI }} \\
& \underbrace{\mathrm{Ha}^{2} u^{\prime+} \frac{\partial \varphi^{+}}{\partial z^{+}}}_{\text {MHD2 }} \underbrace{-\mathrm{Ha}^{2}\left(\overline{u^{\prime+2}}+\overline{w^{\prime+2}}\right)}_{\text {MHD dissipation }} .
\end{aligned}
$$

Here the new terms MHD1, MHD2, and MHD dissipation are caused by the applied magnetic field. The terms in Eq. (10) are shown in Figs. 5(a) $(\mathrm{Ha}=0), 5(\mathrm{~b})(\mathrm{Ha}=32.5)$, and $5(\mathrm{c})(\mathrm{Ha}=65)$. Although the profile shapes of each term do not change with increasing magnetic field, the absolute values for all terms decrease with increasing magnetic field. The contributions of the three additional MHD terms are very small compared to the other terms.

Figure 6 shows the dissipation terms for the applied magnetic field cases; these were obtained by expanding the data in Fig. 5. The MHD1 and MHD2 terms include relative potential gradient components. MHD2 is larger than MHD1.
All terms at $\mathrm{Ha}=65$ are greater than at $\mathrm{Ha}=32.5$. The peak position of all the terms is at $y^{+}=15$, and the region that corresponds to the peak position of the velocity fluctuation.

Figures 7(a)-7(c) show the streamwise wavelengths of the peak of the premultiplied power spectrum at different distances from the wall. In these figures, the vertical axis is the peak of the premultiplied power spectrum, and the horizontal axis represents the distance from the wall. Both axes are normalized by the channel half-width, and are defined by wall units. The structure scales in Fig. 7(a) are approximately divided into two layers at $y^{+}=200$; this is because the scale at $\mathrm{Ha}=0$ in Fig. 7(b) is markedly changed at $y^{+}=200$. The two layers can be defined as the region near wall region and the channel central region. When a magnetic field is applied, the dominant scale of the structure in the region near the wall becomes larger than when there is no magnetic field. However, the dominant scale of the structure at the channel central region decreases with increasing magnetic field.

The spanwise wavelength of the peak of the premulti-

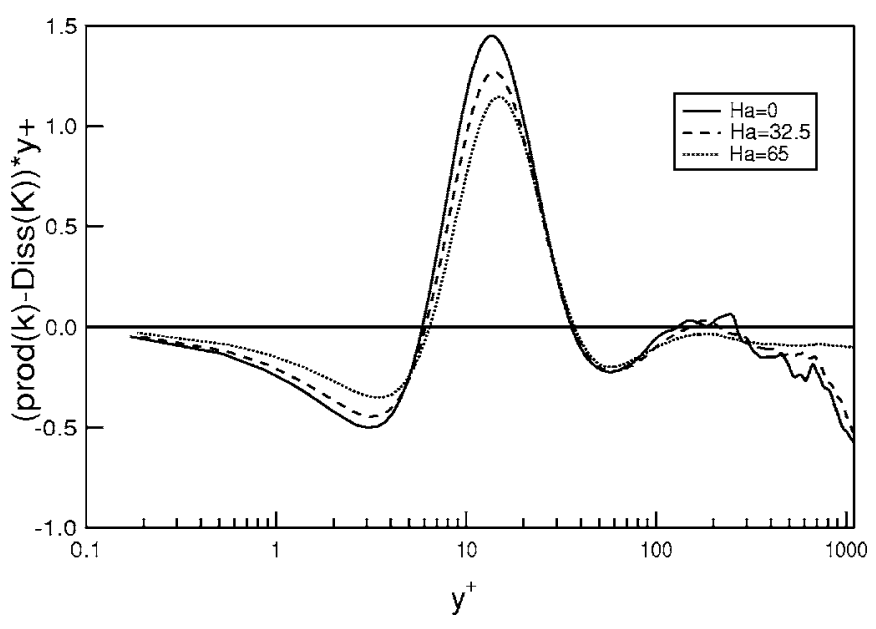

FIG. 9. Difference between production and dissipation. 
(a)
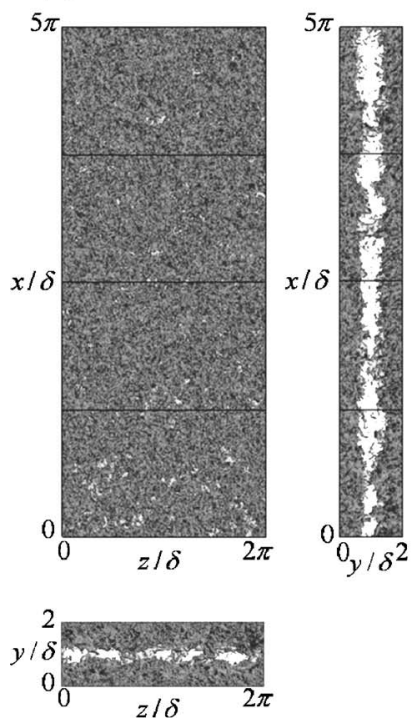

(b)

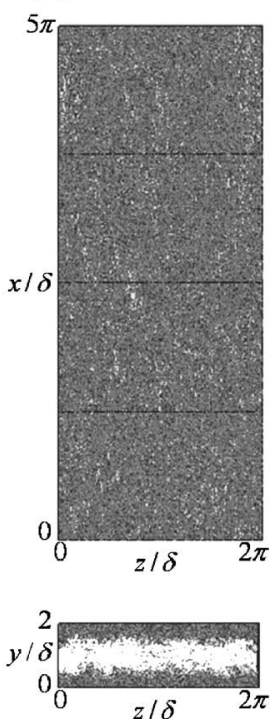

(c)

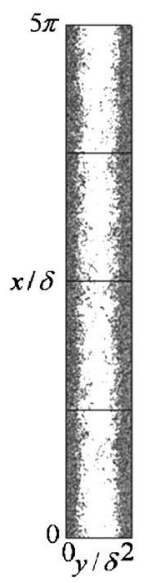

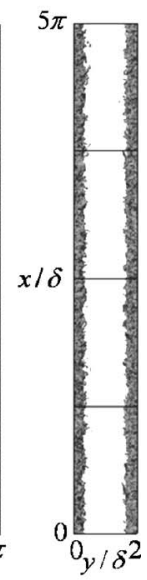

FIG 10. Contour of the second invariant of the velocity gradient tensor; $Q^{+}=0.008$; (a) $\mathrm{Ha}=0$, (b) $\mathrm{Ha}=32.5$, (c) $\mathrm{Ha}=65$. plied power spectrum at different distances from the wall is shown in Figs. 8(a)-8(c). The dominant scale of structures at the region near the wall is almost the same; this is true even when a magnetic field is applied. However, the scale at the channel center decreases. Consequently, when a magnetic field is applied, the primary structure scales at the streamwise and spanwise directions decay at the channel central region; while at the region near the wall, the primary structure scales at the streamwise direction are enhanced and the primary structure scales in the spanwise direction reduced.

In order to explain this mechanism, the integration of the difference between the production and dissipation terms in the turbulent kinetic energy budget is shown in Fig. 9. The profile has both positive and negative values for the production of turbulent energy. For $\mathrm{Ha}=0$ and 32.5, positive regions exist at $6<y^{+}<40$ and at $y^{+}=200$. The first region contributes to the turbulent energy for wall eddies by the production of wall shear. The second region contributes to the energy production of large-scale turbulent structures. For $\mathrm{Ha}=65.0$, there is no positive region for $y^{+}=200$ as this region decays due to the increase in the magnetic field resulting in the reduction of the Reynolds shear stress near the wall, and resulting in the increase of the mean velocity gradient (Fig. 3).

To examine the phenomenon of changing dominant turbulent structure scales owing to magnetic field, the turbulent structures are visualized in Figs. 10-13 without the reduction of grid numbers. The visualization is made using AVS/ Express PCE (Parallel Cluster Edition) of KGT, since the data are huge; one dataset is $6.4 \mathrm{~GB}$. The visualization volume is divided into four regions in the streamwise direction by using PC cluster machines with four processors. The nominal visualization lengths for the computational volumes are $5 \pi \delta, 2 \delta$, and $2 \pi \delta$ in the $x, y$, and $z$ directions. When the lengths are normalized by $\nu$ and $u \tau$, the full visualized volume is $\left(L x^{+}=17592, L y^{+}=2240, L z^{+}=7037\right)$ at $\mathrm{Ha}=0$. (a)
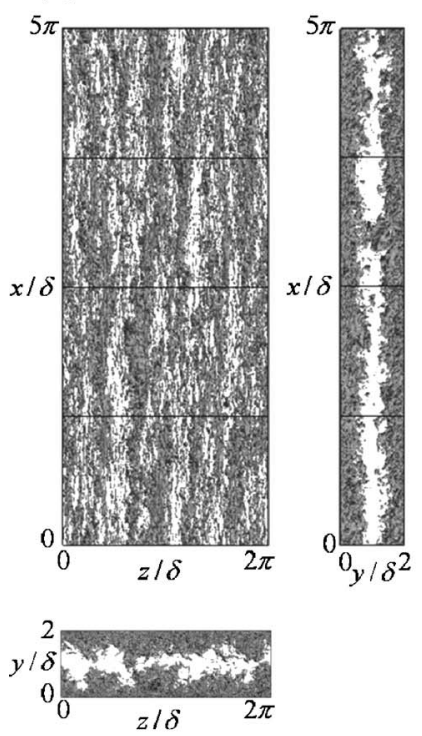

(b)
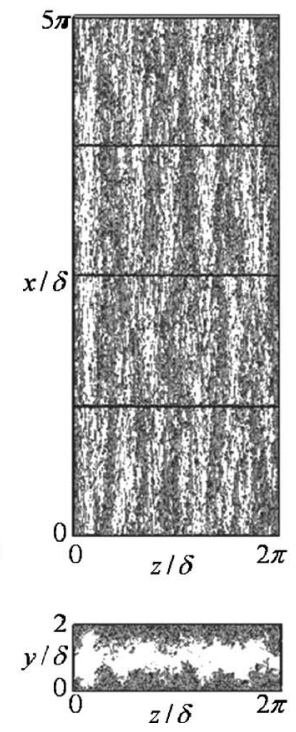

(c)

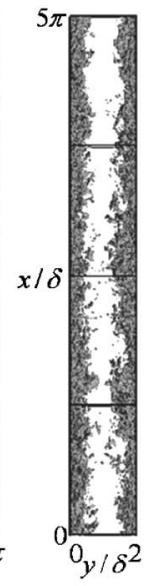

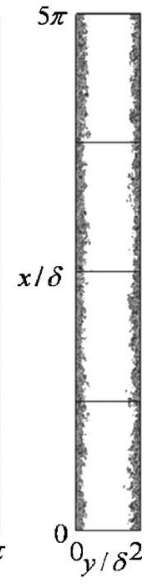

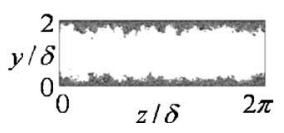

FIG. 11. Contour of streaky structure; $u^{+}<-3$; (a) $\mathrm{Ha}=0$, (b) $\mathrm{Ha}=32.5$, (c) $\mathrm{Ha}=65$. 
(a)

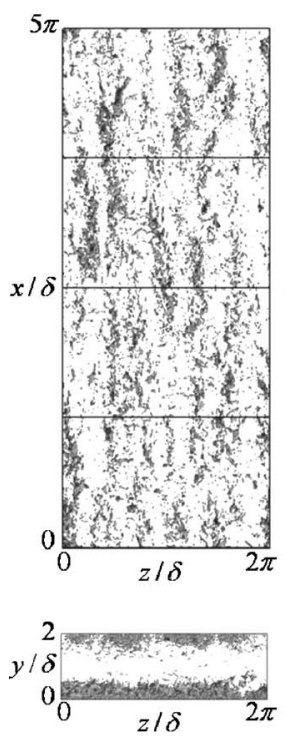

(b)
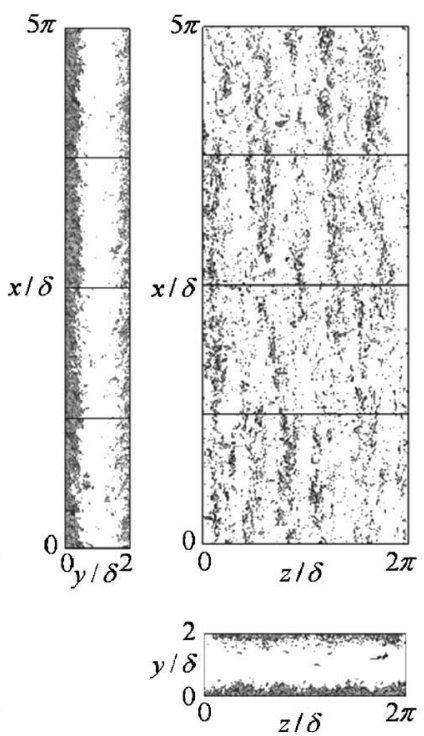

(c)
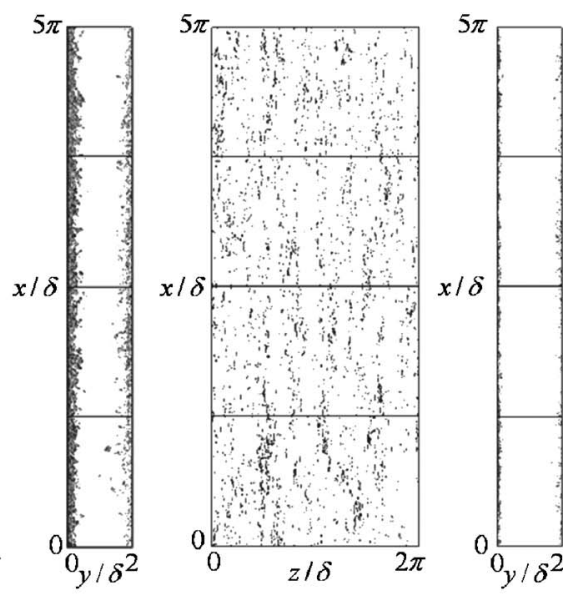

FIG. 12. Contour of sweep; $u^{+} v^{+}<-3$; (a) $\mathrm{Ha}=0$, (b) $\mathrm{Ha}=32.5$, (c) $\mathrm{Ha}=65$.
Figures 10(a)-10(c) show 3D contour surfaces for the second invariant of the velocity gradient tensor. The vortices are identified by isosurfaces of the second invariant of the velocity gradient tensor $\left(Q^{+}=0.008\right)$. Each figure shows three view points, top view, side view, and back-end view. At $\mathrm{Ha}=0$, several vortical structures are located over the whole region. Especially, large-scale vortices appear in the channel center region. However, when the magnetic field is increased, the vertical structures decay at the channel center, leaving only the ones located near the wall regions.

Streaky structures are shown in Figs. 11(a)-11(c). Large streaky structures with many streaks appear in the channel center in the streamwise direction. At $\mathrm{Ha}=0$, large streaky structures with width larger than 1000 exist, and are located away from the wall. This coincides with the spanwise wavelength of the peak of the premultiplied power spectrum in Fig. 7(a). A few merged large streaks elongated in the channel center away from the wall are shown in Fig. 11(a). The characteristic size of the large streaky structures in the streamwise direction is larger than half the channel width. Almost all large structures are almost all located at $y^{+}>200$ [see Fig. 7(a)], corresponding to the wake region in the mean velocity profile. On the other hand, at $\mathrm{Ha}=32.5$ and 65 , the large-scale motion at the channel center decreases in Fig. 11(c). It is evident that the turbulent intensities in Fig. 4 decrease at the channel center owing to the applied magnetic field. Moreover, streaky structures with widths of about 100 wall units for $\mathrm{Ha}=65$ appear near to the wall region. This scale is the primary scale near the wall region and is also seen for low Reynolds number. Thus, near-wall structures and large-scale structures exist at high Reynolds number; the latter ones decreases with the application of a magnetic field and the former ones remain.

To investigate the Reynolds stress contribution to turbulent production, quadrant analysis with four parts including ejection and sweep is performed. Robinson ${ }^{19}$ revealed the (a)
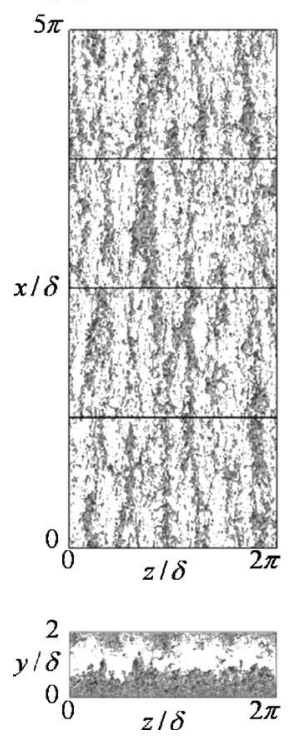

(b)
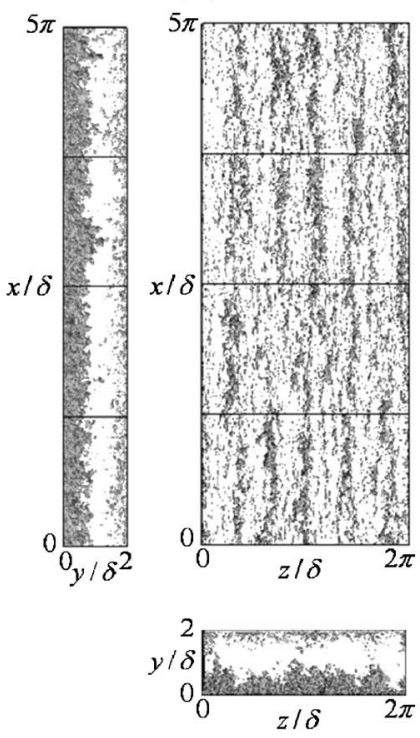

(c)

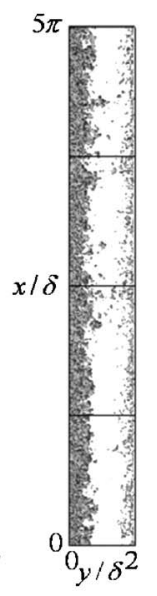

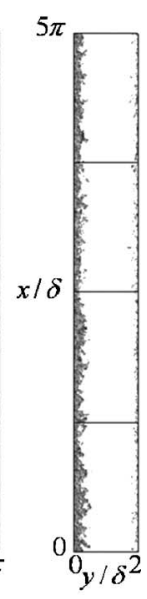

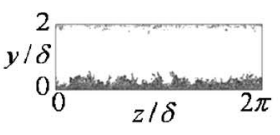

FIG. 13. Contour of ejection; $u^{+} v^{+}<-3$; (a) $\mathrm{Ha}=0$, (b) $\mathrm{Ha}=32.5$, (c) $\mathrm{Ha}=65$. 
existence of near-wall vertical structures and associated ejection/sweep events. Moreover, Kasagi et al. ${ }^{20}$ have presented the details of the spatial relationship between nearwall vortices and ejection/sweep. The isosurface of a sweep mode $(u>0, v<0)$ in one of the quadrants is shown in Fig. 12 . Note that the sweep regions visualized are focused on the wall side at $y / \delta=0$. The other modes at the region on the opposite wall side, for outward interaction $(u>0, v>0)$, are the same. At $\mathrm{Ha}=0$ and 32.5, sweep can be clearly observed from the wall to the channel center. At high-Reynolds number pipe flow, large-scale sweep has also been observed by Satake et $a .^{21}$ However, at $\mathrm{Ha}=65$, sweep regions are limited to regions close to the wall. This is also evidence of the relationship between sweep and Reynolds stress distribution (see Fig. 3).

The isosurface of the ejection mode $(u<0, v>0)$ in one of the quadrants is shown in Fig. 13. Note that the ejection regions visualized are focused on the wall side at $y / \delta=0$ The other modes at the region on the opposite wall side, for inward interaction $(u<0, v>0)$, are the same. The ejection region at the channel center decreases with increasing magnetic field. The scale of ejection in the stream and spanwise directions also decay with increasing magnetic field. It is evident that the causes of the large sweep and ejection are closely related to the mechanism for producing energy (see Fig. 9).

\section{SUMMARY}

DNS for channel flow at high Reynolds number was carried out under a uniform magnetic field. Large scale structures are found to decrease in a transverse magnetic field and vortex structures, streaky structures, sweep, and ejection are found to become smaller at the channel central region. This reduction is caused by a positive contribution to turbulence production takes place in the central region. Evidence for this phenomenon is also seen in the premultiplied power spectra. The wavelengths in both in the $x$ and $z$ directions are shorter than in the non-MHD case. Although the magnetic dissipation terms at $\mathrm{Ha}=65$ are slightly larger than at $\mathrm{Ha}$ $=32.5$, their contribution is small compared to the other terms in the turbulent kinetic energy budget. These results show that the mechanism causing the reduction of large-scale turbulence structures is a reduction in production at the central region due to a decrease in the velocity gradients caused by Hartmann flattening. At high Reynolds number, the turbulence intensities and large-scale structures are found to be reduced.

The findings of this study not only contribute to elucidating the physics of MHD turbulence, but are also useful for understanding how magnetic fields can control turbulent large-scale structures at high Reynolds number.

\section{ACKNOWLEDGMENT}

This work is performed with the support and under the auspices of the NIFS Collaborative Research Program No. NIFS03KTBT002.

${ }^{1}$ M. A. Abdou and the APEX Team, "Exploring novel high power density concepts for attractive fusion systems," Fusion Eng. Des. 45, 145 (1999). ${ }^{2}$ C. P. C. Wonga, S. Malang, M. Sawan, I. Sviatoslavsky, E. Mogahed, S. Smolentsev, S. Majumdar, B. Merrill, R. Mattas, M. Friend, J. Bolin, and S. Sharafat, "Molten salt self-cooled solid first wall and blanket design based on advanced ferritic steel," Fusion Eng. Des. 72, 245 (2004).

${ }^{3}$ C. B. Reed and P. S. Lykoudis, "The effect of a transverse magnetic field on shear turbulence," J. Fluid Mech. 89, 147 (1978).

${ }^{4}$ Y. Shimomura, "Large eddy simulation of magnetohydrodynamic turbulent channel flows under a uniform magnetic field," Phys. Fluids A 3, 3098 (1991).

${ }^{5}$ P. Orlandi, "Drag reduction in turbulent MHD pipe flows," CTR, Proceedings of the Summer Program, 1996, p. 447.

${ }^{6} \mathrm{~S}$. Satake, T. Kunugi, and S. Smolentsev, "DNS of turbulent pipe flow in a transverse magnetic field," J. Turbul. 3, 020 (2002).

${ }^{7}$ S. Satake, T. Kunugi, and S. Smolentsev, "Advances in direct numerical simulation for MHD modeling of free surface flows," Fusion Eng. Des. 61-62, 95 (2002).

${ }^{8}$ D. Lee and H. Choi, "Magnetohydrodynamic turbulent flow in a channel at low magnetic Reynolds number," J. Fluid Mech. 439, 367 (2001).

${ }^{9}$ S. Smolentsev, M. Abdou, N. Morley, A. Ying, and T. Kunugi, "Application of the ' $K$-epsilon' model to open channel flows in a magnetic field," Int. J. Eng. Sci. 40, 693 (2002).

${ }^{10} \mathrm{~S}$. Kenjeres, K. Hanjalic, and D. Bal, "A direct-numerical-simulationbased second-moment close for turbulent magnetohydrodyanmics flows," Phys. Fluids 16, 1229 (2004).

${ }^{11} \mathrm{H}$. Kobayashi, "LES of MHD turbulent channel flows with a local SGS model based on coherent structures," CTR Annual Research Briefs, 2005, p. 235.

${ }^{12}$ R. J. Adrian, C. D. Meinhart, and C. D. Tomkins, "Vortex organization in the outer region of the turbulent boundary layer," J. Fluid Mech. 422, 1 (2000).

${ }^{13}$ J. C. Del Alamo, J. Jimenez, P. Zandonade, and R. D. Moser, "Scaling of the energy spectra of turbulent channels," J. Fluid Mech. 500, 135 (2004).

${ }^{14}$ S. Satake, T. Kunugi, K. Takase, Y. Ose, and N. Naito, "Large-scale structures of turbulent shear flow," Lect. Notes Comput. Sci. 2858, 468 (2003).

${ }^{15} \mathrm{H}$. Abe, H. Kawamura, and Y. Matsuo, "Surface heat-flux fluctuations in a turbulent channel flow up to $\operatorname{Re} \tau=1020$ with $\operatorname{Pr}=0.025$ and 0.71 ," Int. J. Heat Fluid Flow 25, 404 (2004).

${ }^{16}$ K. Iwamoto, N. Kasagi, and Y. Suzuki, "Dynamical roles of large-scale structures in turbulent channel flow," Proceedings of the Computational Mechanics, WCCM VI in Conjunction with APCOM'04, 5-10 September 2004, Beijing, China, MS022-174.

${ }^{17}$ S. Satake and T. Kunugi, "DNS of turbulent channel and pipe flow with high Reynolds number," Proceedings of the 3rd International Symposium on Turbulence and Shear Flow Phenomena, Sendai, 2003, p. 479.

${ }^{18}$ E. C. Brouillette and P. S. Lykoudis, "Magneto-fluid-mechanic channel flow I. Experiment," Phys. Fluids 10, 995 (1967).

${ }^{19}$ S. K. Robinson, "The kinematics of turbulent boundary layer structure," NASA TM-103859 (1991).

${ }^{20}$ N. Kasagi, Y. Sumitani, Y. Suzuki, and O. Iida, "Kinematics of the quasicoherent vortical structure in near-wall turbulence," Int. J. Heat Fluid Flow 16, 2 (1995).

${ }^{21}$ S. Satake, T. Kunugi, and R. Himeno, "High Reynolds number computation for turbulent heat transfer in a pipe flow," Lect. Notes Comput. Sci. 1940, 514 (2000). 